\title{
In Situ Swelling Behavior of Chitosan-Polygalacturonic Acid/Hydroxyapatite Nanocomposites in Cell Culture Media
}

\author{
Rohit Khanna, Kalpana S. Katti, and Dinesh R. Katti \\ Department of Civil Engineering, North Dakota State University, Fargo, ND 58105, USA \\ Correspondence should be addressed to Kalpana S. Katti, kalpana.katti@ndsu.edu
}

Received 12 February 2010; Revised 22 March 2010; Accepted 22 March 2010

Academic Editor: Shanfeng Wang

Copyright (C) 2010 Rohit Khanna et al. This is an open access article distributed under the Creative Commons Attribution License, which permits unrestricted use, distribution, and reproduction in any medium, provided the original work is properly cited.

In this work, we report the evaluation of molecular and mechanical characteristics of in situ degradation behavior of chitosanpolygalacturonic acid/hydroxyapatite (Chi-PgA-HAP) nanocomposite films. The degradation behavior is studied using Fourier Transform Infrared spectroscopy (FTIR), Atomic Force Microscopy (AFM), and modulus mapping techniques for up to 48 days of soaking in cell culture media. The surface molecular structure of media soaked samples changes over the course of 48 days of soaking, as indicated by significant changes in phosphate vibrations $\left(1200-900 \mathrm{~cm}^{-1}\right)$ indicating formation of apatite. ChitosanPolygalacturonic acid polyelectrolyte complexes (PECs) govern the structural integrity of Chi-PgA-HAP nanocomposites and FTIR spectra indicate that PECs remain intact until 48 days of soaking. In situ AFM experiments on media soaked samples indicate that soaking results in a change in topography. AFM topographic images indicate that swelling proceeds in an inhomogeneous fashion during the initial soaking period up to 8 days and then, occurs in a more gradual fashion during the longer soaking periods. In situ modulus mapping experiments on soaked samples that measure properties by probing $\sim 1-3 \mathrm{~nm}$ of surface represent the pure elastic properties of surface of soaked samples. Elastic moduli of $\sim 4 \mathrm{GPa}$ are obtained from the surfaces. These represent the nanoscale elastic responses of proteins adsorbed on Chi-PgA-HAP nanocomposites or elastic properties of swollen polymeric structures. Modulus mapping results of soaked samples indicate that nanoscale elastic modulus decreases by $\sim 2 \mathrm{GPa}$ over a long exposure to cell culture media (48 days). Overall results suggest that as water enters the Chi-PgA-HAP sample, surface molecular interactions in Chi-PgA-HAP structure occur that can result in swelling, as seen in topographical changes of soaked samples and small changes in nanoscale mechanical properties were also observed.

\section{Introduction}

Recent developments in design of novel polymeric biomaterials have allowed researchers to confront many of the challenges dealing with the design of novel biomaterials. The tremendous potential of biodegradable polymers for tissue engineering and medical devices results from their biocompatibility, ease in processing, and capability of controlled degradation in response to biological environment. Candidate materials for bone tissue engineering include natural polymers (chitosan, collagen, hyaluronan, fibrin), synthetic polymers (polycaprolactone, polylactic acid, polyglycolic acid, and their copolymers) and inorganic materials (tricalcium phosphate, hydroxyapatite). Also, the natural biodegradable and biofunctional biopolymer, chitosan, has been considered as a potential candidate material for numerous biomedical applications including controlled drug release $[1,2]$, wound dressing [3], and more recently, for tissue engineering application [4-13]. Chitosan $[\beta(1,4)$ linked 2-amino-2-deoxy-D glucan] is a cationic polysaccharide obtained by $\mathrm{N}$-acetylation of chitin. Chitosan provides improved cell attachment, since the polysaccharide backbone of chitosan is structurally similar to glycoaminoglycans, the major component of extracellular matrix of cartilage and bone [14]. Due to cationic character of chitosan, it interacts with a number of polyanions and has been used in preparation of various polyelectrolyte complexes with natural polyanions such as alginic acid, dextran sulphate, heparin, pectin, and xanthan $[15,16]$. Another natural biodegradable biopolymer, polygalacturonic acid (PgA), is a water-soluble polyanionic polysaccharide, consisting of a linear chain of D-galacturonic acid units with $\alpha(1-4)$ glycosidic linkages. This is a principal component of pectin that exists in cellular walls of plants. Pectin is extensively 
used in food industry as a gelling agent and widely used in the production of jams and jellies, confectionary products and for stabilization of yogurts [17] and drug production such as for antidiarrheic, detoxicants and as protectors of gastrointestinal tract $[18,19]$.

Appropriate mechanical property of bone tissue engineering scaffold is one of the key factors in selection and design of biomaterials, which may limit the independent use of natural chitosan/pectin-based materials. Chitosan, has been reported to possess improved biocompatibility, mechanical strength, hard tissue regeneration and tailored degradation kinetics by incorporation of hydroxyapatite (HAP) [20, 21] and gelatin [20, 22, 23], alginate [24], and so forth. By employing electrostatically complementary nature of chitosan and PgA and by adopting a biomimetic synthesis route, we designed and developed novel chitosan-PgAhydroxyapatite (Chi-PgA-HAP) nanocomposites [25-27] which exhibited a substantial improvement in mechanical properties. Our FTIR results demonstrated that strong interfacial interactions in Chi-PgA-HAP structures are believed to be the key factors in mechanical property improvement [25-27]. Biocompatibility results demonstrated that human osteoblasts (CRL 11732) generated mineralized bone nodules on Chi-PgA-HAP fibrous nanocomposites without using any differentiating media suggesting that Chi-PgAHAP substrates are osteoinductive and provide an appropriate microenvironment for cell organization and tissue regeneration [27].

For suitability of Chi-PgA-HAP nanocomposites for bone tissue engineering, their degradation behavior needs to be evaluated. Ideally, material degradation rate should match the rate of tissue regeneration, while generating nontoxic degradation products. Both chitosan and pectin are well known to be enzymatically degradable and water resistant $[18,19,28,29]$. Several investigations on enzymatic degradation of chitosan (with varying degree of deacetylation) for human use have been carried out in lysozyme [30-34], since it is found in human body fluid, including serum (concentration 4-13 mg/L) and tears ( 450-1230 mg/L) [35]. Chitosan-pectin membranes have been reported to swell considerably in water and their swelling characteristics depend on $\mathrm{pH}$, temperature, ionic strength, and degree of polyelectrolyte complex formation $[18,29,36]$. Swelling behavior of chitosan-pectin membranes is an attractive property for drug delivery systems [37, 38]. In situ swelling behavior of Chitosan-PgA-HAP nanocomposites in terms of change in surface properties has not been investigated. Here, we present the in situ swelling behavior of Chi-PgA-HAP nanocomposite films over the soaking period of 48-day in cell culture media. In situ swelling experiments include the evaluation of topography, chemistry and nanomechanical properties of soaked 2D substrates in a fully hydrated state. $2 \mathrm{D}$ substrates have been utilized due to their relative ease as compared to 3D scaffolds. In situ AFM experiments were performed in cell culture media at a temperature of $37^{\circ} \mathrm{C}$, to obtain the topography of soaked samples.

Recently, there is much interest in obtaining the mechanical properties of surface of biomaterial substrates. As cells attach onto the substrate, they respond to stiffness of the substrate which further modulates their biological functions $[37,38]$. Modulus mapping is a surface nanomechanical characterization technique that enables the measurement of purely elastic properties of material surface by applying extremely shallow displacements $(\sim 2-3 \mathrm{~nm})$. Modulus mapping technique has been recently used to obtain the surface elastic properties of dentin-enamel junction [39] and biomimetic nanohydroxyapatite particles [40]. However, this technique has not been used till now to evaluate the purely elastic properties of constituents of soaked samples under fully immersed conditions. In the present work, in situ modulus mapping experiments were designed and elastic properties of soaked samples were obtained in fully submerged conditions (cell culture media; $37^{\circ} \mathrm{C}$ ) by applying extremely shallow displacements $(\sim 2-3 \mathrm{~nm})$. Molecular structures of both dry and soaked specimen was investigated by Fourier Transform Infrared spectroscopy.

\section{Materials and Methods}

2.1. Film Preparation. HAP nanoparticles were prepared by wet precipitation route from sodium phosphate $\left(\mathrm{Na}_{2} \mathrm{HPO}_{4}\right.$; J. T. Baker), and calcium chloride $\left(\mathrm{CaCl}_{2} ; \mathrm{EM}\right.$ Sciences $)$ by maintaining $\mathrm{pH}$ at 7.44. The detailed processing procedures have been discussed elsewhere [41]. Chitosan (MW 190,000, $>85 \%$ deacetylation) and polygalacturonic acid (MW 25000) were obtained from Sigma-Aldrich chemicals. Chitosan and PgA solutions were prepared in deionized water at a concentration of $1 \mathrm{~g} / 100 \mathrm{~mL}$. The $\mathrm{pH}$ of chitosan solution was adjusted by adding acetic acid and that of PgA solution was adjusted by adding $\mathrm{NaOH}$. The $\mathrm{pH}$ of resulting solutions was adjusted to 4.5. Chitosan-PgA solution was prepared by adding chitosan solution dropwise to PgA solution followed by sonication for 3 minutes. For synthesis of Chi-PgA-HAP nanocomposite, HAP nanoparticles $(1 \mathrm{~g})$ were suspended in $100 \mathrm{~mL}$ of deionized water and sonicated for 1 hour to obtain a uniform dispersion of nanoparticles. Subsequently, sonicated HAP nanoparticles were mixed with sonicated Chitosan-PgA solution and further sonicated for 90 seconds to obtain a homogeneous solution. Films of two compositions were prepared (Chi-PgA-10\% HAP and Chi-PgA$20 \%$ HAP) on TCPS (Tissue Culture Polystyrene) petridishes by solvent evaporation at room temperature under clear-air laboratory environment. For making films, Chi-PgA-HAP solution was diluted to $1: 10$ ratio with deionized water and $3 \mathrm{~mL}$ of resulting solution was air-dried on tissue culture polystyrene petridishes. Both the nanocomposite substrates were found to be biocompatible and Chi-PgA-20\% HAP composition was selected for soaking study.

2.2. Soaking Experiments. All the samples were UV sterilized for 1 hour and subsequently soaked in $5 \mathrm{~mL}$ of cell culture media for 1, 2, 8, 24, and 48-day, respectively, under standard incubator conditions $\left(37^{\circ} \mathrm{C}, 5 \% \mathrm{CO}_{2}\right)$. Cell culture media was prepared by adding DMEM (Dulbecco's modified eagle medium; Hyclone), supplemented with $2.5 \mathrm{mM}$ l-glutamine (without phenol red), 10\% FBS (fetal bovine serum; ATCC) and $1 \%$ antibiotic (G418; J R Scientific Inc.). At the end of 
predetermined time, films samples were taken out of incubator, and washed twice with PBS and immediately taken for obtaining molecular structures, topography and elastic properties using FTIR, AFM and nanoindentation analyses, respectively. For making molecular structure-topography correlations, all the samples were subjected to same soaking conditions for carrying out AFM/FTIR/nanoindentation analyses. Culture media was replaced every 3 days.

2.3. FTIR Experiments. FTIR experiments were carried out using a Nicolet 850 FT-IR spectrometer with a $\mathrm{KBr}$ beam splitter using reflectance accessory ( $45^{\circ}$ angle of incidence). FTIR in a specular reflectance mode was used to detect the surface molecular structures from within $1 \mu \mathrm{m}$ depth. Spectra were collected in the range of $4000-500 \mathrm{~cm}^{-1}$ at a spectral resolution of $4 \mathrm{~cm}^{-1}$. Film samples of chitosan, $\mathrm{PgA}$ and Chi-PgA-HAP nanocomposites were prepared on the clean gold substrates and allowed to dry overnight. Airdried samples were used for soaking studies. FTIR spectra were obtained on soaked samples which were air-dried before data acquisition. For all test conditions, at least 3 scans were recorded on different regions on the samples and representative spectra were analyzed. A gold-coated metal plate was used to collect the background spectrum.

2.4. In Situ AFM Experiments Using Multimode Heater. Topography of dry and soaked samples was characterized by Atomic Force Microscope (multimode AFM: Veeco Metrology Group, Santa Barbara, CA), equipped with a Nanoscope IIIa controller and J-type piezo scanner. The equipment has $z$-axis resolution of around $0.5 \mathrm{~A}^{\circ}$. Silicon nitride cantilever probes (Model: NP-S20) with pyramidal tips of $\sim 20 \mathrm{~nm}$ radius of curvature and a nominal stiffness of $0.06 \mathrm{~N} / \mathrm{m}$ were used for imaging under contact mode in clean-air laboratory environment with 40\%-50\% relative humidity. For performing in situ AFM experiments in fully immersed conditions, Multimode low temperate heater from Veeco Metrology (Santa Barbara, CA) was used. It enabled in situ AFM analyses of soaked samples in a controlled liquid environment. Major components of the system include a fluid cell, a heating element, Thermal Applications Controller (TAC), and spacer block. For a detailed description of components available for Multimode Scanning Probe Microscopes, AFM technical support note 392 can be referred. The sample temperature was set and maintained at $37^{\circ} \mathrm{C}$ by regulating the heating element and tip heater voltage with the help of Thermal Applications Controller (TAC). The whole sample-fluid-tip was thermally equilibrated for at least 20 minutes to enable the tests to be performed in a stable and controlled environment (cell culture media; $37^{\circ} \mathrm{C}$ ). For investigating the major changes in substrate topography of soaked samples, scans of $100 \mu \mathrm{m} \times 100 \mu \mathrm{m}$ were obtained on both dry and soaked samples. AFM topography of large areas of soaked samples were obtained by taking at least 10 scans of $100 \mu \mathrm{m} \times 100 \mu \mathrm{m}$ area on different regions on the sample surface and representative AFM topography images were further analyzed.

2.5. In Situ Modulus Mapping Technique. A Hysitron triboscope nanomechanical instrument (Minneapolis, MN) equipped with nanoscope IIIa controller (Veeco Metrology, Santa Barbara, CA) was used to test the dry and soaked samples with a Berkovich (three-sided pyramid; a 100$200 \mathrm{~nm}$ tip radius) diamond indenter fluid tip. The fluid tip with a longer steel shaft allows a liquid level up to 3$4 \mathrm{~mm}$ to be maintained above the sample surface and also ensures the machine calibration in the liquid. In order to avoid any meniscus forces, the tip was fully submerged in the liquid prior to testing. Modulus mapping technique was used to obtain in situ modulus maps on soaked samples in cell culture fluid and at a temperature of $37^{\circ} \mathrm{C}$ to simulate incubator conditions. Detailed description of principles of modulus mapping technique, its formulation and application can be found elsewhere $[39,42]$. In force modulation mode, a quasistatic force of $3 \mu \mathrm{N}$ was superimposed by a $2 \mu \mathrm{N}$ sinusoidal force at a frequency of $200 \mathrm{~Hz}$. A total of $256 \times$ 256 tests were done to obtain a modulus map in only 10-15 minutes.

For testing in liquid environment, a special fluid cell was designed by using O-rings (9/16" O.D. $\left.\times 3 / 8^{\prime \prime} \times 3 / 32^{\prime \prime}\right)$ glued onto $15 \mathrm{~mm}$ steel discs. Samples $(0.5 \mathrm{~cm} \times 0.5 \mathrm{~cm})$ were glued and kept inside the fluid cell and then, flushed with liquid level up to 3-4 mm for testing in fully submerged conditions. The whole tip-sample-fluid assembly was then heated and maintained to $37^{\circ} \mathrm{C}$ using Multimode heater as described in Section 2.4. Before data acquisition, the entire setup was allowed to equilibrate for at least 20 minutes. Before starting modulus mapping experiment, the tip was wiped with acetone soaked tissue wipes to remove any ions/salts which may have adsorbed from the previous measurements. Tip calibration was done using a standard quartz sample with known elastic modulus. Extreme care was taken to keep the samples under the wet conditions before the in situ testing to avoid the effect of drying on nanomechanical measurements.

All the AFM, modulus mapping and FTIR experiments were performed using on least 3 samples for each set of test conditions. All the tests were repeated at least twice to account for repeatability and reproducibility of test measurements.

\section{Results and Discussion}

3.1. Molecular Nature of Surfaces of Soaked Chi-PgA-HAP Nanocomposite Samples. FTIR spectroscopy was used in a reflection mode to characterize the surface molecular structure of soaked samples. Figure 1 shows the FTIR spectra of dry and soaked Chi-PgA-HAP samples within the range $4000-500 \mathrm{~cm}^{-1}$. The band positions and their assignments have been summarized in Tables 1, 2, and 3. The band at $1666 \mathrm{~cm}^{-1}$, as seen in Figure 1, is a characteristic vibration of $\mathrm{N}$-acetylated chitin and is assigned to Amide I band. As samples are soaked in cell culture media, proteins can get adsorbed onto the sample surface due to electrostatic interactions of PECs with proteins [4]. Amide I band from carbonyl stretching in protein structure is observed at 1666 $\mathrm{cm}^{-1}$ band position, which also overlaps with amide I band from chitosan structure. The band at $1622 \mathrm{~cm}^{-1}$ is attributed to asymmetric stretching vibration of dissociated 


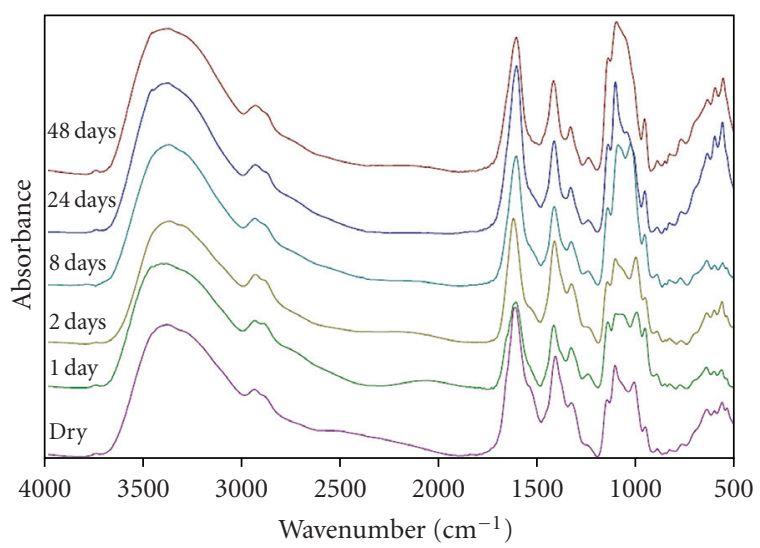

FIGURE 1: FTIR spectra obtained on dry and soaked Chi-PgA-HAP nanocomposite film samples after 1, 2, 8, 24 and 48-day, respectively in the regions $4000-500 \mathrm{~cm}^{-1}$.

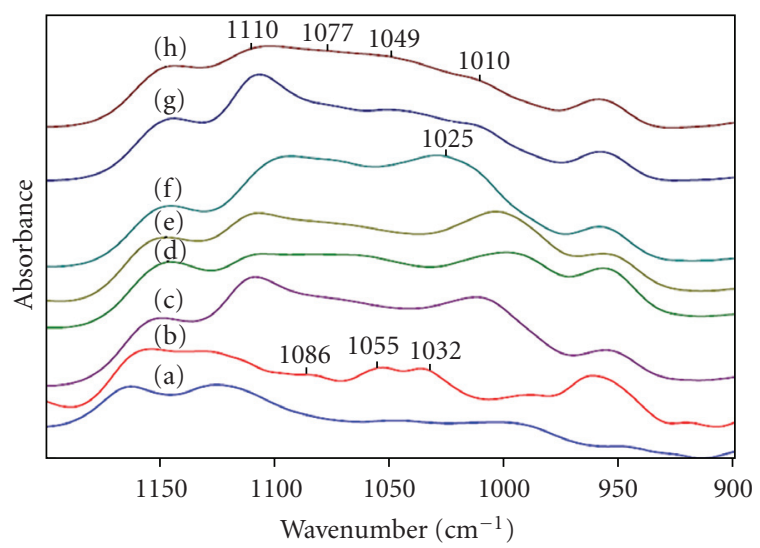

Figure 2: FTIR spectra of dry chitosan (a), dry PgA (b), dry Chi-PgA-HAP nanocomposites (c) and soaked Chi-PgA-HAP nanocomposite film samples over $1,2,8,24$ and 48-day (d-h), respectively in the regions $1200-900 \mathrm{~cm}^{-1}$.

carboxylate $\left(\mathrm{COO}^{-}\right)[26,43]$. This band shifts slightly to lower wavenumber, to $1605 \mathrm{~cm}^{-1}$ in soaked samples, which indicates that the change in environment of Chi-PgAHAP structure through its interaction with water, possibly through hydrogen bonds with Chi-PgA-HAP structure. As water enters the Chi-PgA-HAP sample which contains polyelectrolyte complexes with $-\mathrm{NH}_{3}^{+},-\mathrm{COO}^{-}$ions, surface structure of Chi-PgA-HAP structure can get modified. In such a case, swelling can occur due to mutual repulsion between positively/negatively charged species along with interactions of these charges species with water. Swelling characteristics of chitosan-pectin have been reported to be modified by preparing the samples at varying $\mathrm{pH}$ [44].

PgA-HAP interactions were also affected as a result of soaking, as revealed in $1100-1000 \mathrm{~cm}^{-1}$ region of FTIR spectra of soaked samples (Figure 2). PgA interacts with HAP through dissociated carboxylate groups. As carboxylate groups carry negative charge, calcium ions in HAP can act as binding sites for PgA-HAP complexes [26]. To investigate PgA-HAP interactions, phosphate regions were carefully observed for both dry and soaked samples, as shown in Figure $2(\mathrm{a}-\mathrm{h})$. Figure $2(\mathrm{a}, \mathrm{b})$ shows the FTIR spectrum obtained from dry chitosan and dry PgA film, respectively. Bands observed at $1086 \mathrm{~cm}^{-1}, 1055 \mathrm{~cm}^{-1}$, and $1032 \mathrm{~cm}^{-1}$ in PgA (Figure 2(b)), originate from skeletal vibrations involving $\mathrm{C}-\mathrm{O}$ stretching, which are characteristic of saccharide structure of PgA [18]. Comparing FTIR spectrum of PgA (Figure 2(b)) with that of dry Chi-PgA-HAP (Figure 2(c)), bands originating from $-\mathrm{C}-\mathrm{O}-\mathrm{C}-$ glycosidic linkages overlap with bands from phosphate regions and occur as a broad band $\left(1100-1000 \mathrm{~cm}^{-1}\right)$ in Figure 2(c). Some of the characteristic bands of HAP structure, that is, asymmetric PO stretching vibrations in HAP structure occur at $1113 \mathrm{~cm}^{-1}$, $1070 \mathrm{~cm}^{-1}$, and $1009 \mathrm{~cm}^{-1}$ regions can be clearly observed in dry Chi-PgA-HAP samples. Significant changes in band positions in phosphate regions $\left(1200-900 \mathrm{~cm}^{-1}\right)$ were not observed throughout the soaking duration. However, significant changes in shape of bands in phosphate regions $\left(1200-900 \mathrm{~cm}^{-1}\right)$ in soaked samples, over a soaking duration of 48-day indicate that interactions in PgA-HAP structure changes as a result of soaking. Comparing spectrum of PgA (Figure 2(b)), dry Chi-PgA-HAP (Figure 2(c)), and pure HAP [45] with that of 48-day soaked sample (Figure 2(h)), characteristic shape of phosphate regions observed on 48 soaked sample matched with that observed in HAP structure. It appears, that as soaking proceeds, more HAP nanoparticles may get exposed onto the surface of soaked samples. Similar observations of exposed HAP particles were made in AFM topography image of 48-day soaked samples, as discussed in the next section. There can be another possibility of apatitic growth on soaked film samples, which accounted for the visibility of characteristic bands of HAP structure on 48-day soaked Chi-PgA-HAP sample.

Overall, FTIR spectroscopy results on soaked samples indicate that the degradation of Chi-PgA-HAP nanocomposite films was not severe in cell culture medium until 48 -day of soaking. There were apparent changes in surface chemistry of soaked Chi-PgA-HAP substrates as compared to dry substrates. Severe degradation can be expected on dissociation of Chi-PgA polyelectrolyte complexes (PECs) and can also be due to breaking of -C-O-C- glycosidic linkages in Chitosan/PgA structure. Spectral changes in both Chi-PgA-HAP complexes and -C-O-C- glycosidic linkages were not observed in soaked samples. It can be due to the fact that both chitosan and PgA have been reported to be enzymatically degradable $[19,30,31]$ and degrade primarily through lysozyme-mediated hydrolysis. Moreover, fully deacetylated chitosan has low degradation rates due to inability of hydrolytic enzymes to penetrate the crystalline microstructures. Slow degradation characteristics in our study can be due to high deacetylation degree of chitosan $(>85 \%)$ and maintenance of structural integrity of PECs in cell culture media.

3.2. AFM Topographic Characterization. Topographical signatures of swelling/degradation were investigated by carrying out systematic in situ AFM experiments in hydrated environment using the Multimode heater, as described in 


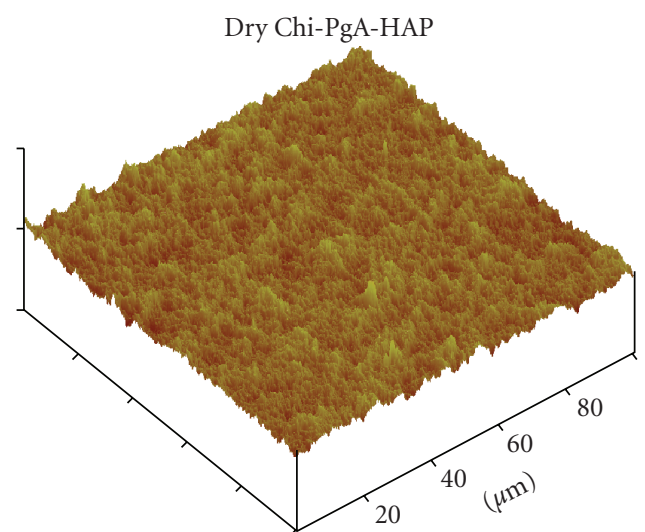

$x 20 \mu \mathrm{m} / \mathrm{div}$

z $3000 \mathrm{~nm} / \mathrm{div}$

(a)

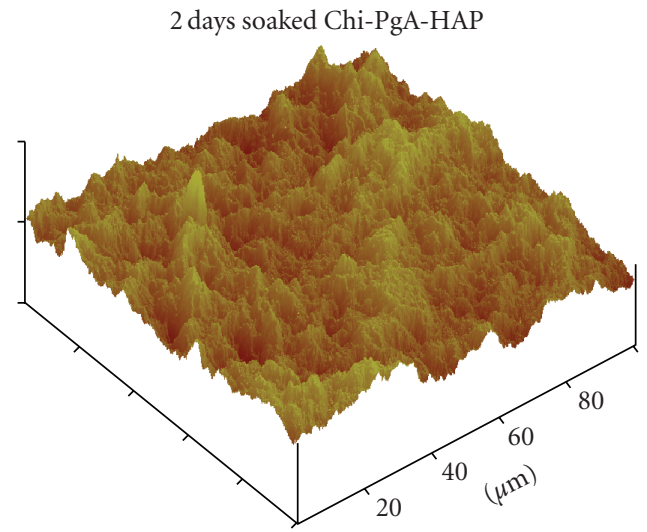

$x 20 \mu \mathrm{m} / \mathrm{div}$

z $3000 \mathrm{~nm} / \mathrm{div}$

(c)

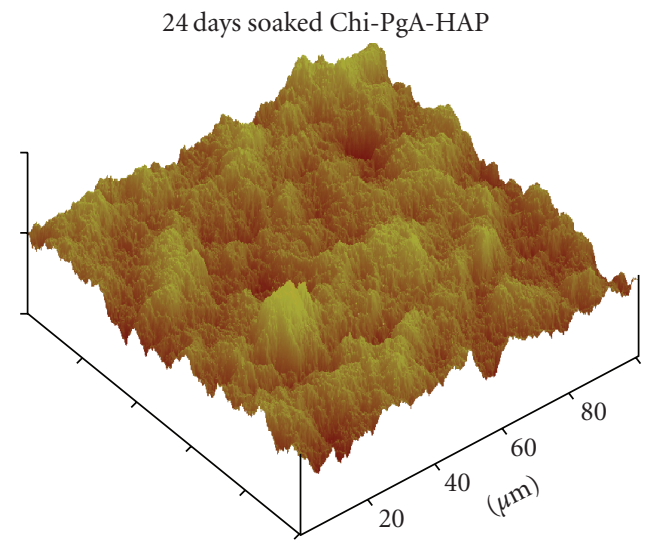

$x 20 \mu \mathrm{m} / \mathrm{div}$

z $3000 \mathrm{~nm} / \mathrm{div}$

(e)

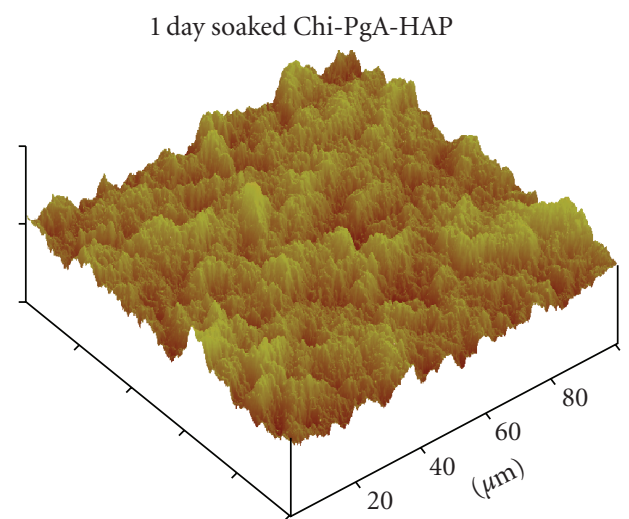

$x 20 \mu \mathrm{m} / \mathrm{div}$

$z 3000 \mathrm{~nm} / \mathrm{div}$

(b)

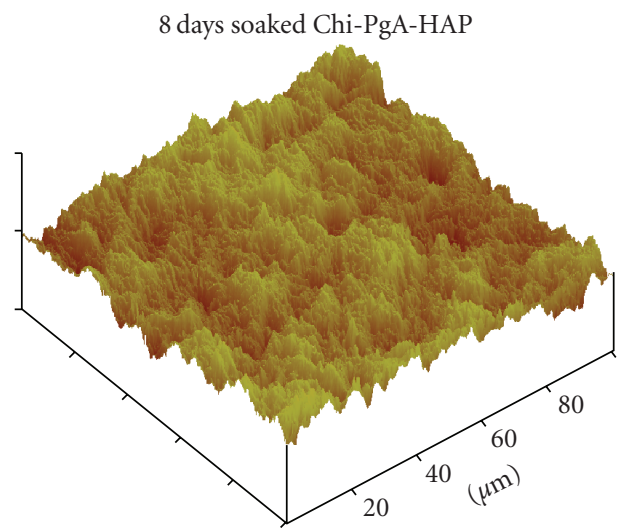

$x 20 \mu \mathrm{m} / \mathrm{div}$

$z 3000 \mathrm{~nm} / \mathrm{div}$

(d)

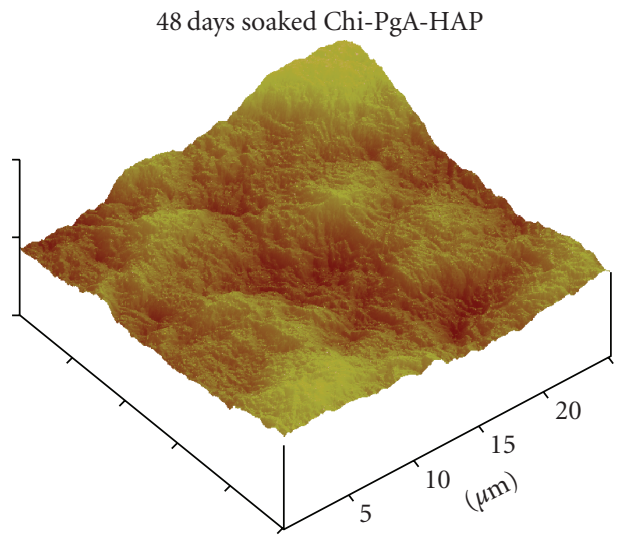

$x 5 \mu \mathrm{m} / \mathrm{div}$

z $2999.999 \mathrm{~nm} / \mathrm{div}$

(f)

Figure 3: AFM 3D topographic images of dry (a) and soaked Chi-PgA-HAP samples for 1, 2, 8, 24, and 48-day, respectively (b)-(f). Topographic changes are evident after soaking in cell culture media. Topographic features (ridges, valleys, and pits) appear in a random fashion during the initial soaking duration up to 8 days, and then, more gradual changes are observed during the longer soaking period up to 48 -day, as seen in topographic images (e, f). 
TABLE 1: Band assignment of dry and soaked Chi-PgA-HAP films for $3380-2860 \mathrm{~cm}^{-1}$ regions.

\begin{tabular}{|c|c|c|c|c|c|c|}
\hline \multirow{2}{*}{ Band assignment } & \multicolumn{6}{|c|}{ Band position as per the testing conditions } \\
\hline & Dry & 1 day & 2 days & 8 days & 24-day & 48-day \\
\hline Asymmetric N-H stretching & 3386 & 3377 & 3365 & 3365 & 3368 & 3360 \\
\hline symmetric $\mathrm{N}-\mathrm{H}$ stretching & 3290 & 3284 & 3292 & 3288 & not observed & not observed \\
\hline Asymmetric $\mathrm{C}-\mathrm{H}$ stretching & 2931 & 2936 & 2933 & 2932 & 2934 & 2933 \\
\hline Symmetric C-H stretching & 2878 & 2878 & 2874 & 2878 & 2866 & 2869 \\
\hline
\end{tabular}

TABLE 2: Band assignment of dry and soaked Chi-PgA-HAP films for $1660-1240 \mathrm{~cm}^{-1}$ regions.

\begin{tabular}{|c|c|c|c|c|c|c|}
\hline \multirow{2}{*}{ Band assignment } & \multicolumn{6}{|c|}{ Band position as per the soaking conditions } \\
\hline & Dry & 1 day & 2 days & 8 days & 24-day & 48-day \\
\hline Amide I & 1666 & 1665 & Not observed & 1666 & 1665 & 1665 \\
\hline Asymmetric $\mathrm{COO}^{-}$stretching & 1622 & 1605 & 1624 & 1604 & 1605 & 1603 \\
\hline $\mathrm{NH}_{3}^{+}$deformation & 1534 & 1532 & 1534 & 1533 & 1534 & 1539 \\
\hline symmetric $\mathrm{COO}^{-}$stretching & 1414 & 1417 & 1418 & 1420 & 1416 & 1421 \\
\hline \multirow{2}{*}{$\mathrm{C}-\mathrm{H}$ bending vibration of ring } & 1330 & 1331 & 1329 & 1328 & 1332 & 1336 \\
\hline & 1242 & 1245 & 1237 & 1244 & 1243 & 1246 \\
\hline
\end{tabular}

Section 2.4. Representative AFM topographic images of dry and soaked film samples are shown in Figure 3. Topography of soaked samples was investigated for 1, 2, 8, 24 and 48-day, respectively. For investigating the major changes in substrate topography at the microscale, large scan sizes $(100 \mu \mathrm{m} \times 100 \mu \mathrm{m})$ were obtained on both dry and soaked regions. Figure 3(a) shows a smooth 3D topography of dry Chi-PgA-HAP nanocomposite. Further, significant changes in topography were observed after soaking in cell culture medium, as indicated in Figures 3(b)-3(f). After 1 day of soaking, a rough corrugated appearance as indicated by the uneven distribution of sharp ridges and valleys in the microstructure (Figure 3(b)) is observed. Figure 3(c) shows the $3 \mathrm{D}$ topography after 2 days of soaking. Ridges appear to grow in height and were observed to have nonuniform distribution over the entire scan area. After 8 days of soaking, some of sharp ridges appear to decrease in height or flatten out and more valleys or grooves were observed (Figure 3(d)). The ridges appear to flatten out and take the shape of circular features (bright spots), were observed to be unevenly distributed over the microstructure.

Soaking experiments were carried out for longer periods to observe the topographical changes of Chi-PgA-HAP samples over a long term exposure. Figure 3(e) indicates the 3D topography of 24-day soaked substrate. As seen in AFM topographic image, deep grooves and pits appear to be present over the entire area. In the literature, swelling characteristics of chitosan and pectin based materials have been commonly reported in terms of the water absorption and water retention $[18,28,30,32,46]$. Our AFM results demonstrate that topography changes dramatically upon soaking in cell culture media ( 1 day) which can be due to swelling of Chi-PgA-HAP biodegradable polymer nanocomposite. Irregularities in topographic features indicate that swelling profiles are site-specific, depending on the access of water into the polymer structures. Due to hydrophilic nature of Chi-PgA-HAP nanocomposite, diffusion of water into the matrix of nanocomposite is faster than degradation, which can result in swelling of the Chi-PgA-HAP nanocomposite prior to degradation. Swelling appears to proceed in a nonuniform fashion during the initial soaking period up to 8 days as indicated by dramatic changes in ridges and valleys in AFM topography images. Sizes and shapes of circular features also appear to be distorted and the irregular shaped larger pits appear to be formed by the coalescence of smaller pits, as revealed by more diffused patterns of light and dark regions in the AFM topographic image of 48-day soaked sample (Figure 3(f)).

The topographic changes can occur due to change in molecular interactions as demonstrated in FTIR spectra of soaked samples (Figure 1). Representative 2D AFM topographic images $(100 \mu \mathrm{m} \times 100 \mu \mathrm{m})$ were taken for dry (Figure 4(a)), 8 days (Figure 4(b)) and 48-day (Figure 4(c)) soaked nanocomposite samples to correlate the topographic observations with the chemistry of soaked samples. As seen in Figure 4(a), bundles of Chi-PgA fibers were observed to be uniformly distributed in the microstructure. HAP nanoparticles were not observed on the surface of dry samples. The average size of HAP particles has been reported to be $\sim 50 \mathrm{~nm}$ and the results have been reported elsewhere [40]. Complementary observations on molecular structures of samples were made using FTIR experiments, that is, characteristic shape of bands in phosphate regions (as observed in FTIR spectrum on pure HAP sample [45]) was not observed on dry samples which indicates that HAP particles are not exposed on the surface of dry sample and detection depth for specular reflectance was not sufficient to detect the HAP nanoparticles lying underneath the sample surface. After 8 days of soaking in cell culture media, dot-like features appear to be exposed on the surfaces of soaked sample, as 
TABLE 3: Band assignment of dry and soaked Chi-PgA-HAP films for $1150-500 \mathrm{~cm}^{-1}$ regions.

\begin{tabular}{lcccccc}
\hline \multirow{2}{*}{ Band assignment } & \multicolumn{5}{c}{ Band position as per the soaking conditions $\left(\mathrm{cm}^{-1}\right)$} \\
\hline C-O stretching in $v(\mathrm{C}-\mathrm{O}-\mathrm{C})$ & 1155 & 1150 & 1154 & 1151 & 1149 & 48 -day \\
$\nu_{3}$ asymmetric PO stretching & 1113,1070 & 1107,1061 & 1110,1067 & 1102 & $1107,1072,1043$ & $1110,1077,1049$ \\
$\nu_{1}$ symmetric PO stretching & 1009,954 & 996,953 & 1001,952 & 1021,956 & 1009,956 & 1010,960 \\
P-O $(\mathrm{H})$ stretching & 894 & 895 & 894 & 895 & 896 & 896 \\
O-H bending in HAP structure & 645 & 645 & 645 & 644 & 643 & 645 \\
$\nu_{4}$ O-P-O bending & 569 & 569 & 568 & 566 & 563 & 564 \\
\hline
\end{tabular}

seen in AFM topographic image (Figure 4(b)). FTIR results on 8 days soaked samples indicate that major changes in molecular structures were observed in the phosphate regions and dot-like features were observed in AFM topographic image. Combining the results of both AFM and FTIR experiments for dry and soaked samples, it appears that changes in surface molecular structures of sample caused the appearance of dot-like features on the sample surface which can be the clusters of HAP nanoparticles. FTIR spectrum taken on 48 days samples exhibited the characteristic shape of phosphate bands which match closely with that observed in case of pure HAP samples[45]. AFM 2D height image (Figure $4(\mathrm{c})$ ) on 48 days soaked sample indicate that $\sim 80 \%$ of the scan area is covered with HAP nanoparticles, which suggests that as soaking proceeds, molecular interactions in Chi-PgA-HAP structures change (phosphate regions; Figure 2), thereby, exposing more HAP nanoparticles to surface of soaked samples and these observations were not made in dry Chi-PgA-HAP samples (both AFM images \& FTIR results).

\subsection{Nanoscale Elastic Properties by In Situ Modulus Mapping} Technique. In situ elastic properties of soaked samples were obtained in presence of cell culture media and at a temperature of $37^{\circ} \mathrm{C}$ (using Multimode heater) using modulus mapping technique. The description of test procedures is given in Section 2.4. Figure 5(a) shows the modulus map of a scan area of $10 \mu \mathrm{m} \times 10 \mu \mathrm{m}$ on surface of dry ChiPgA-HAP nanocomposite film. In modulus map image, lighter color corresponds to lower elastic moduli ( $\sim \mathrm{GPa})$ due to relatively softer polymer phase, that is, Chi-PgA phase. Darker color corresponds to higher elastic moduli $(\sim 80 \mathrm{GPa})$, which indicate the elastic responses from the stiffer hydroxyapatite phase or from the regions having a very thin polymer layer. A combined elastic response $(\sim 10$ $30 \mathrm{GPa}$ ) from both the polymer and HAP phase is indicated by mixed color patches observed in the modulus map image. The composite elastic modulus can be obtained from the surfaces of biomimetic nanohydroxyapatite, as reported in our prior work [40]. The variation in modulus values along the scan length of $10 \mu \mathrm{m}$ indicates the local distribution of constituents in the microstructure (Figure 5(b)).

Figures 6(a)-6(d) show the modulus data of samples soaked in cell culture media for 1, 2, 8, and 48-day, respectively. Representative line scans were selected from modulus maps obtained as per each soaking experiment conditions, and modulus values were plotted as function of position. In situ elastic moduli of 3.5-8 GPa have been obtained from the surfaces of soaked substrates for upto 2 days of soaking. These are the nanoscale elastic responses of the individual swollen constituents on the surfaces of ChiPgA-HAP substrate. An observed scatter in modulus values within $4-8 \mathrm{GPa}$ is consistently observed during the initial soaking duration (Figures 6(a) and 6(b)). Over the longer soaking duration (Figures 6(c) and 6(d)), less scatter in moduli was observed and moreover, nanoscale modulus is observed to decrease by $\sim 2 \mathrm{GPa}$.

Elastic modulus in the range of 2-4 GPa [47] has been measured from the gold nanoparticles functionalized with bovine serum albumin and streptavidin using nanoindentation technique (load $=5 \mu \mathrm{N}$ ). The elastic modulus of $\sim 3$ $4 \mathrm{GPa}$ measured using modulus mapping technique (load $=$ $5 \mu \mathrm{N})$ can result from the nanoscale elastic response from the proteins adsorbed on the surface of soaked samples, which also match closely with that reported in literature. Protein adsorption on Chi-PgA-HAP nanocomposites is more likely due to its hydrophilic nature. Specifically, chitosan's cationic nature allows various electrostatic interactions with negatively charged species such as proteins, anionic glycoaminoglycans [4]. Chi-PgA-HAP structure with mainly $\mathrm{NH}_{2},-\mathrm{COOH}$ and $-\left(\mathrm{COO}^{-} \mathrm{NH}_{3}^{+}\right)$complexes can interact with proteins and modify the surfaces. Performing in situ elastic property measurement in cell culture fluid and at $37^{\circ} \mathrm{C}$ can give the nanoscale properties of proteins adsorbed on Chi-PgA-HAP substrates in their natural environment. However, in a modulus mapping technique, it is not easy to identify the elastic responses of individual phases, that is, proteins or surface sites without proteins, due to a potential overlap in the modulus values of proteins and sites of soaked substrate. Similar experiments were done on non-degradable tissue culture polystyrene (TCPS) samples which have a flat topography and uniform protein adsorption. In our modulus mapping data on TCPS samples, modulus values of 3-5 GPa have been observed under the similar soaking conditions. In the absence of any topography effect, these modulus values represents purely elastic responses of proteins (unpublished results).

Further, additional experiments were done on soaked but air dried samples in clear air laboratory environment to see the effect of dehydration from the soaked polymer 


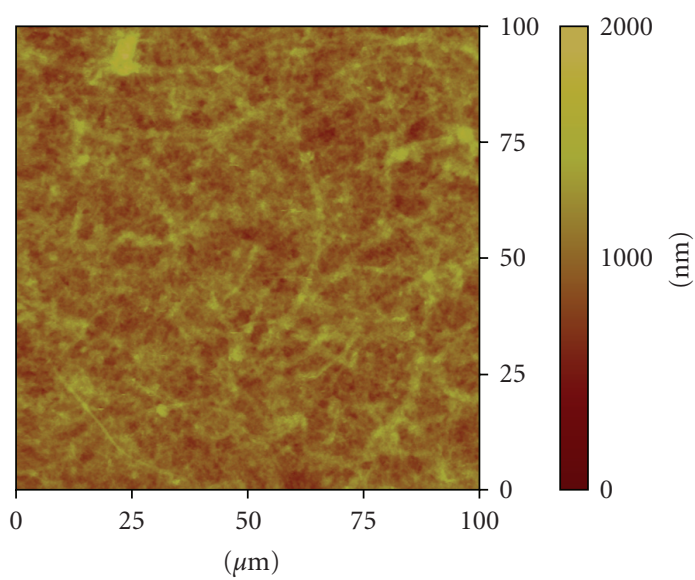

(a) Dry Chi-PgA-HAP nanocomposite

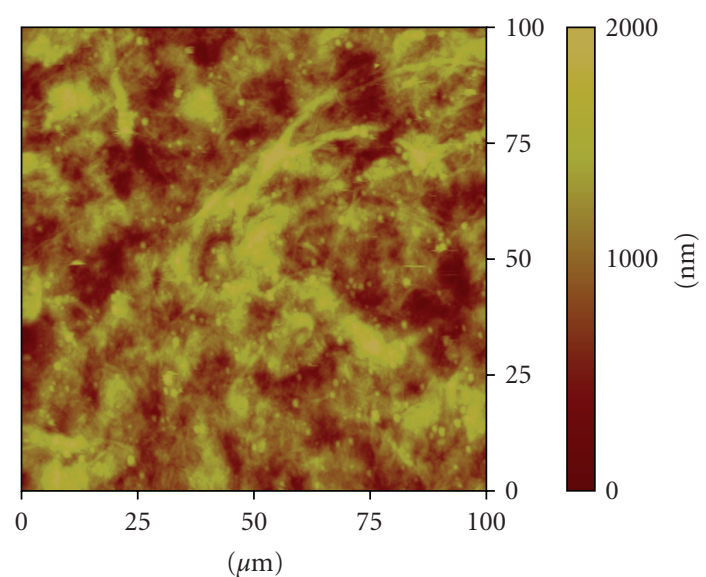

(b) 8 days soaked Chi-PgA-HAP nanocomposite

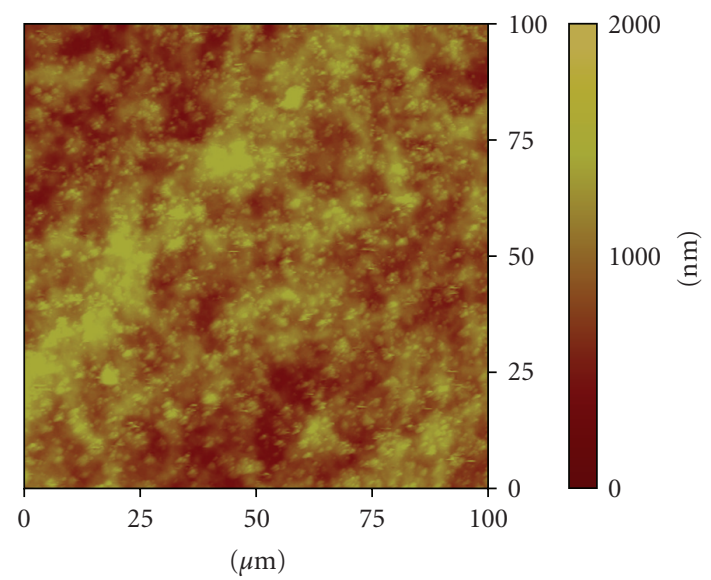

(c) 48-day soaked Chi-PgA-HAP nanocomposite

FIgure 4: AFM topography image indicating the distribution of bundles of Chi-PgA fibers in the Chi-PgA-HAP nanocomposite; HAP nanoparticles are barely discernible in AFM topography image of dry sample. (a) Topographic variations and HAP nanoparticles are more commonly observed in AFM topography image of sample after 8 days of soaking. (b) $\sim 80 \%$ of scan area appears to be covered with HAP nanoparticles in the AFM topographic image obtained on 48-day soaked sample (c).

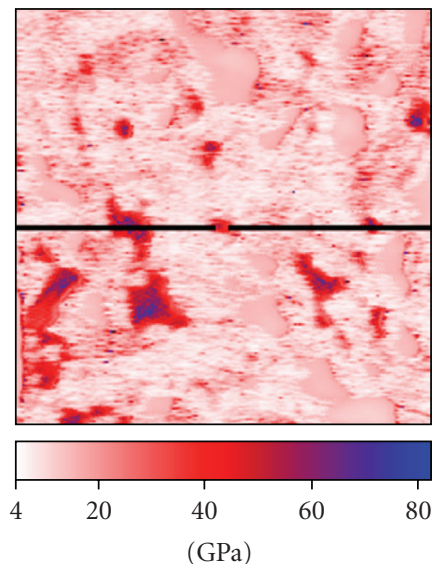

(a)

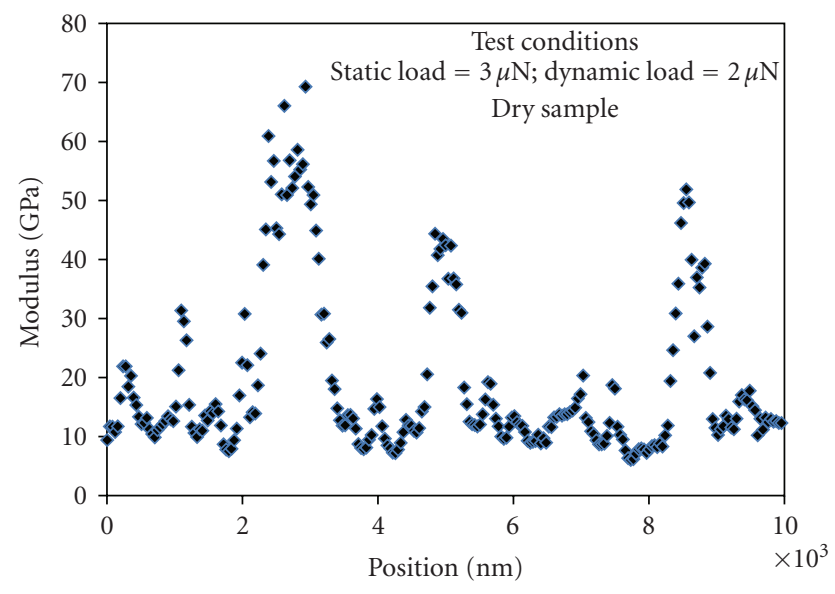

(b)

Figure 5: Modulus map of dry Chi-PgA-HAP nanocomposite $(10 \mu \mathrm{m} \times 10 \mu \mathrm{m})$ (a) and modulus data along the line of $10 \mu \mathrm{m}$ length (b). High modulus values are due to stiffer HAP phase and lower modulus values are due to soft polymer phases. 


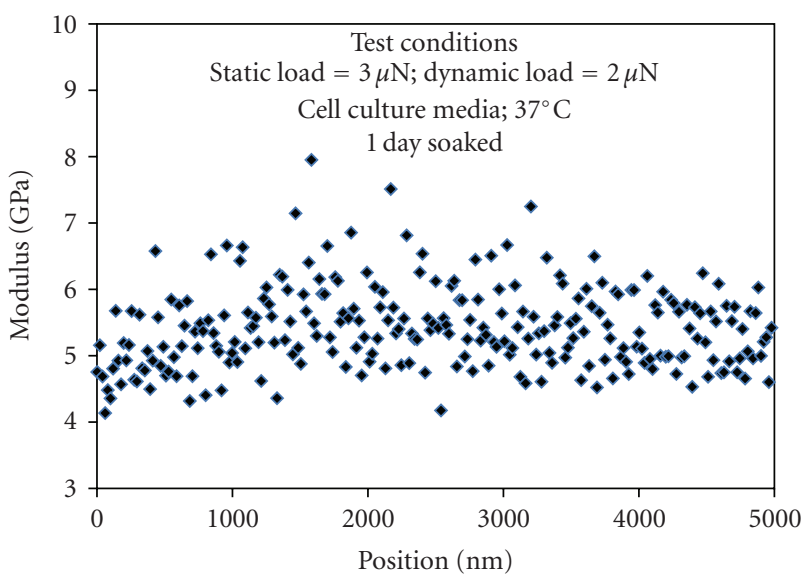

(a)

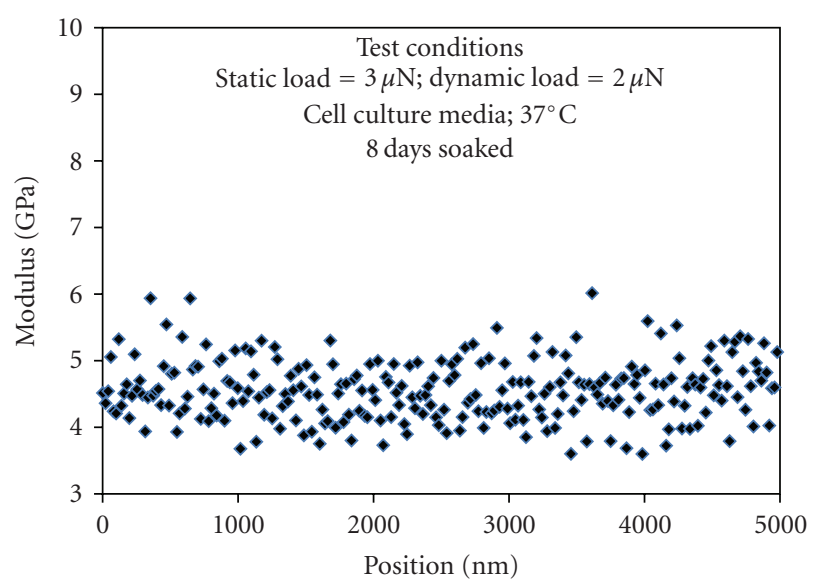

(c)

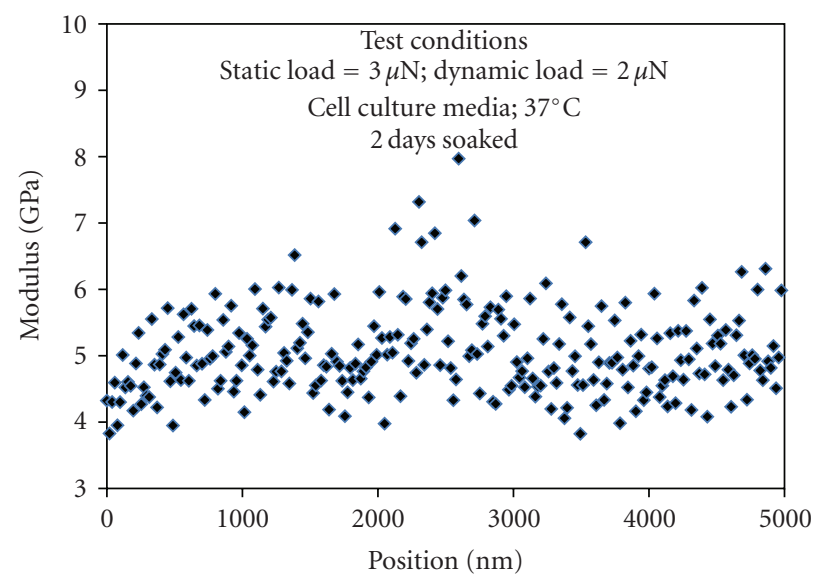

(b)

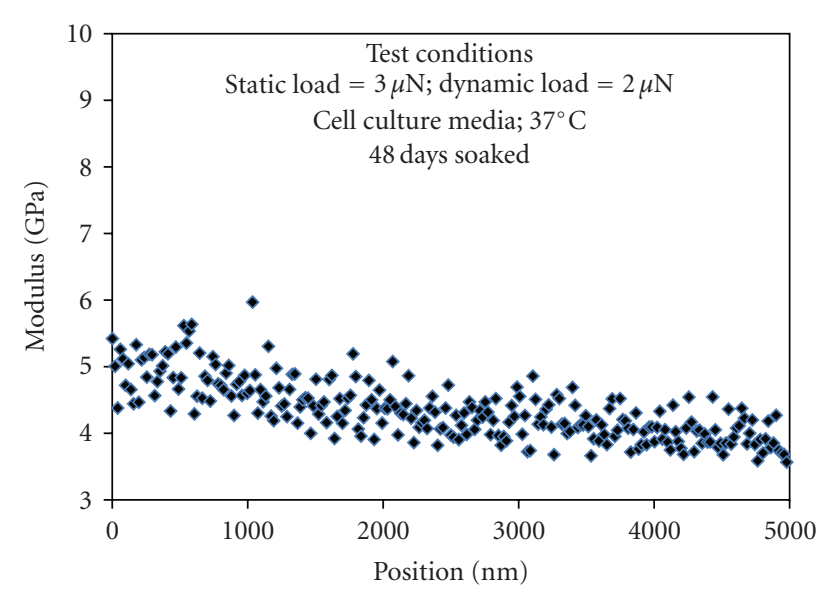

(d)

FIGURE 6: In situ modulus mapping results of 1-(a), 2-(b), 8-(c), and 48-(d) day soaked Chi-PgA-HAP samples. Moduli versus position plots indicate the nanoscale elastic responses of surfaces of soaked samples.

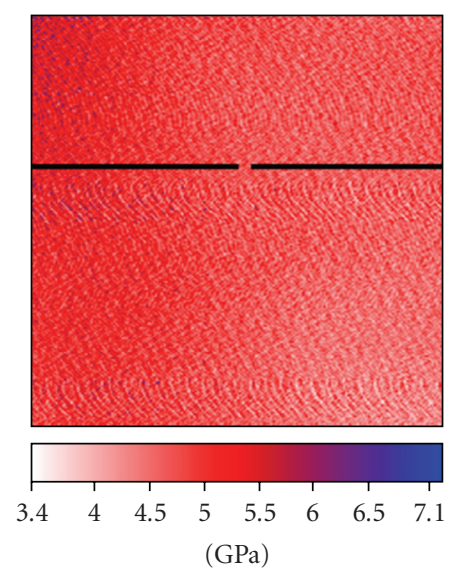

(a)

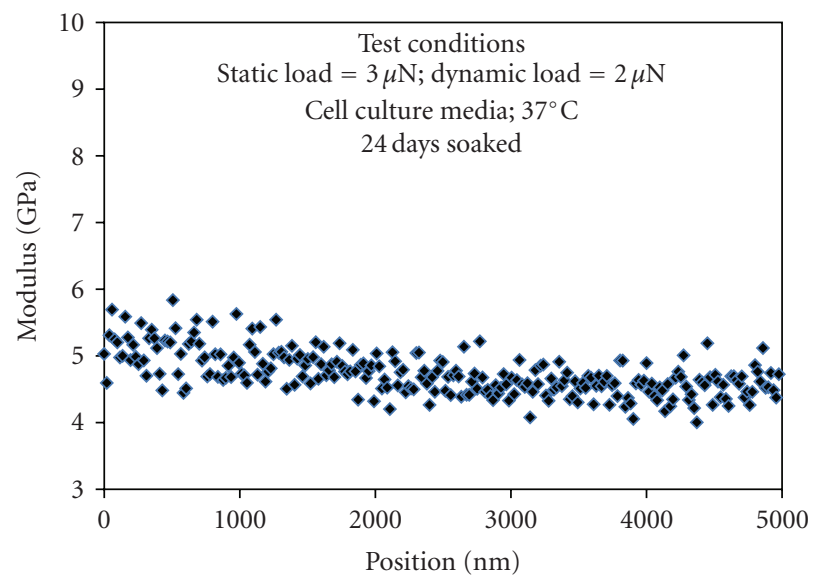

(b)

Figure 7: In situ modulus mapping results of 24-day soaked Chi-PgA-HAP samples. Modulus map indicates that there is not significant variation in surface elastic properties of soaked samples (a); modulus versus position plot indicates the mean modulus values of $\sim 5 \mathrm{GPa}$ (b). 


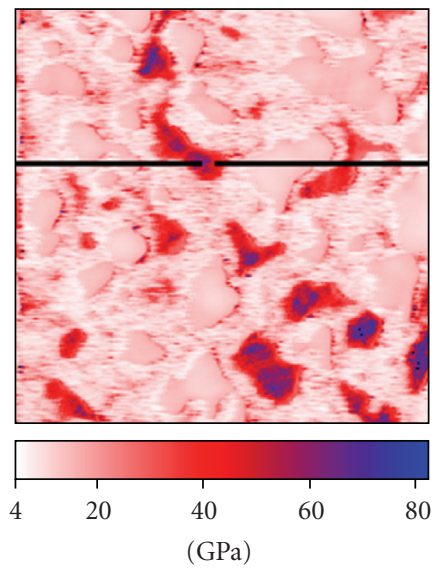

(a)

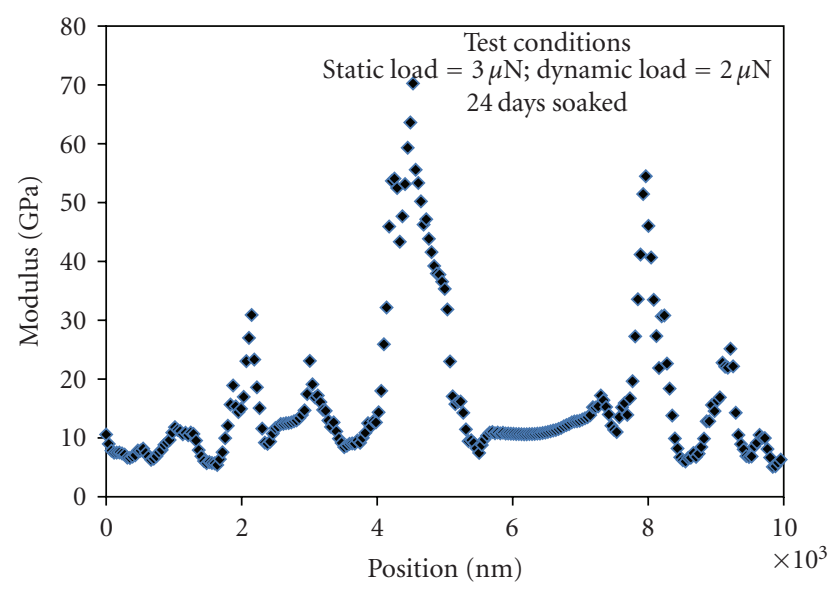

(b)

Figure 8: Modulus mapping results of 24-day soaked (but dried) Chi-PgA-HAP sample and tested in air. Chi-PgA-HAP samples. Modulus map indicates that high moduli in the range of 4-80 GPa are observed on surface of dried samples. (a) Modulus versus position plot indicates the elastic responses of the surfaces of 24-day soaked sample.

nanocomposite samples. Selected results are presented herein for modulus mapping experiments on 24-day soaked samples and tested in a fully hydrated state and unhydrated (dried) environment. Figure 7(a) indicates the modulus map obtained after 24-day of soaking and tested in a hydrated state (cell culture media; $37^{\circ} \mathrm{C}$ ). Modulus values of $4-6 \mathrm{GPa}$ were observed with a mean value of $\sim 5 \mathrm{GPa}$ (Figure $7(\mathrm{~b})$ ). Figure 8(a) indicates the modulus map obtained after 24day of soaking, and completely dried in air, and then, tested in clean-air laboratory environment. Modulus data were plotted as a function of position over a length of $10 \mu \mathrm{m}$ (Figure 8(b)). Modulus values in the range of 4-80 GPa were observed. Significantly large difference in modulus values observed by testing under the hydrated and unhydrated environment can result from dehydration effects from the surface of the swollen polymer specimen which makes its surface more stiff. Effect of sample dehydration on nanomechanical properties is less important for hard materials like metals, ceramics and some hard tissues. However, soft and spongy polymers (e.g., ChiPgA-HAP biodegradable polymer nanocomposites) which exhibit swelling characteristics, have entrapped water in their structures. Hence, testing such samples in a dry environment can severely affect the mechanical properties, as seen in our modulus mapping results. It has been shown that even in case of hard tissue like bone, upon dehydration, hardness can be increased by $17 \%-30 \%$ and modulus by $15 \%-50 \%[48$, 49]. Therefore, it is important that in situ nanomechanical mesurements are carried out in a liquid environment to get in situ elastic properties of water-swollen polymeric structures. Our results suggest that modulus mapping is a promising technique to evaluate the purely elastic properties of proteins and that of soaked substrates.

\section{Conclusions}

The in situ swelling behavior of novel Chi-PgA-HAP nanocomposites is characterized by FTIR, AFM and mod- ulus mapping technique. FTIR results demonstrated that molecular structure of soaked samples changes over the course of 48-day of soaking in cell culture media, as indicated by significant variations in phosphate vibrations. As water enters the Chi-PgA-HAP sample, interactions in Chi-PgA-HAP structure are modified that can result in swelling. PECs govern the structural integrity in Chi-PgAHAP nanocomposites and FTIR spectra indicated that PECs were still in-tact until 48-day of soaking. In situ AFM experiments on soaked samples indicate that soaking results in a change in topography due to swelling of Chi-PgAHAP substrates. AFM topographic images indicate that swelling proceeds in an inhomogeneous fashion during the initial soaking period up to 8 days and then, occurs in a more gradual fashion during the longer soaking periods. In situ modulus mapping experiments on soaked samples represent the elastic properties of surface of soaked samples. Elastic moduli of $\sim 4 \mathrm{GPa}$ are observed, which may result from nanoscale elastic responses of proteins adsorbed on Chi-PgA-HAP nanocomposites. Modulus mapping results of soaked samples indicate that nanoscale elastic modulus decreases by $\sim 2 \mathrm{GPa}$ over a long exposure to cell culture media (48-day) and there were no major changes in surface mechanical properties over 48-day of soaking in cell culture media.

This work has important implications for tissue engineering. For instance, mostly biodegradable materials are used in the fabrication of tissue engineering scaffolds. Biodegradable materials can undergo physic chemicalmechanical changes under the wet conditions, as also indicated by our study. It is now understood that cells do respond to such physico-chemical changes which can also affect cell-biomaterial interactions. In order to correlate the changes in physical properties of degrading substrate to cellular responses (biochemical/biomechanical), a systematic evaluation of in situ material properties of biodegradable materials in nearly physiological environment is required, which merits scientific interest. 


\section{References}

[1] S. Miyazaki, K. Ishii, and T. Nadai, "The use of chitin and chitosan as drug carriers," Chemical \& Pharmaceutical Bulletin, vol. 29, no. 10, pp. 3067-3069, 1981.

[2] K. Aiedeh, E. Gianasi, I. Orienti, and V. Zecchi, "Chitosan microcapsules as controlled release systems for insulin," Journal of Microencapsulation, vol. 14, no. 5, pp. 567-576, 1997.

[3] G. Kratz, C. Arnander, J. Swedenborg, et al., "Heparinchitosan complexes stimulate wound healing in human skin," Scandinavian Journal of Plastic and Reconstructive Surgery and Hand Surgery, vol. 31, no. 2, pp. 119-123, 1997.

[4] S. V. Madihally and H. W. T. Matthew, "Porous chitosan scaffolds for tissue engineering," Biomaterials, vol. 20, no. 12, pp. 1133-1142, 1999.

[5] W.-C. Hsieh, C.-P. Chang, and S.-M. Lin, "Morphology and characterization of 3D micro-porous structured chitosan scaffolds for tissue engineering," Colloids and Surfaces B, vol. 57, no. 2, pp. 250-255, 2007.

[6] Y. Wan, H. Wu, and D. Wen, "Porous-conductive chitosan scaffolds for tissue engineering, 1: preparation and characterization," Macromolecular Bioscience, vol. 4, no. 9, pp. 882-890, 2004.

[7] J. Nakamatsu, F. G. Torres, O. P. Troncoso, M. L. Yuan, and A. R. Boccaccini, "Processing and characterization of porous structures from chitosan and starch for tissue engineering scaffolds," Biomacromolecules, vol. 7, no. 12, pp. 3345-3355, 2006.

[8] A. Wang, Q. Ao, W. Cao, et al., "Porous chitosan tubular scaffolds with knitted outer wall and controllable inner structure for nerve tissue engineering," Journal of Biomedical Materials Research Part A, vol. 79, no. 1, pp. 36-46, 2006.

[9] L. Peng, X. R. Cheng, J. W. Wang, D. X. Xu, and G. Wang, "Preparation and evaluation of porous chitosan/collagen scaffolds for periodontal tissue engineering," Journal of Bioactive and Compatible Polymers, vol. 21, no. 3, pp. 207-220, 2006.

[10] Y. Wan, A. X. Yu, H. Wu, Z. X. Wang, and D. J. Wen, "Porousconductive chitosan scaffolds for tissue engineering II. In vitro and in vivo degradation," Journal of Materials Science: Materials in Medicine, vol. 16, no. 11, pp. 1017-1028, 2005.

[11] H. F. Liu, F. L. Yao, Y. Zhou, et al., "Porous poly (DLlactic acid) modified chitosan-gelatin scaffolds for tissue engineering," Journal of Biomaterials Applications, vol. 19, no. 4, pp. 303-322, 2005.

[12] L. Ma, C. Y. Gao, Z. W. Mao, et al., "Collagen/chitosan porous scaffolds with improved biostability for skin tissue engineering," Biomaterials, vol. 24, no. 26, pp. 4833-4841, 2003.

[13] J. Yang, T. W. Chung, M. Nagaoka, M. Goto, C.-S. Cho, and T. Akaike, "Hepatocyte-specific porous polymer-scaffolds of alginate/galactosylated chitosan sponge for liver-tissue engineering," Biotechnology Letters, vol. 23, no. 17, pp. 13851389, 2001.

[14] E. Khor and L. Y. Lim, "Implantable applications of chitin and chitosan,” Biomaterials, vol. 24, no. 13, pp. 2339-2349, 2003.

[15] S. Dumitriu and E. Chornet, "Inclusion and release of proteins from polysaccharide-based polyion complexes," Advanced Drug Delivery Reviews, vol. 31, no. 3, pp. 223-246, 1998.

[16] C. Peniche and W. Arguelles-Monal, "Chitosan based polyelectrolyte complexes," Macromolecular Symposia, vol. 168, pp. 103-116, 2001.
[17] W. G. T. Willats, P. Knox, and J. D. Mikkelsen, "Pectin: new insights into an old polymer are starting to gel," Trends in Food Science \& Technology, vol. 17, no. 3, pp. 97-104, 2006.

[18] P. Bernabé, C. Peniche, and W. Argüelles-Monal, "Swelling behavior of chitosan/pectin polyelectrolyte complex membranes. Effect of thermal cross-linking," Polymer Bulletin, vol. 55, no. 5, pp. 367-375, 2005.

[19] L. S. Liu, M. L. Fishman, J. Kost, and K. B. Hicks, "Pectinbased systems for colon-specific drug delivery via oral route," Biomaterials, vol. 24, no. 19, pp. 3333-3343, 2003.

[20] F. Zhao, W. L. Grayson, T. Ma, B. Bunnell, and W. W. Lu, "Effects of hydroxyapatite in 3-D chitosan-gelatin polymer network on human mesenchymal stem cell construct development," Biomaterials, vol. 27, no. 9, pp. 1859-1867, 2006.

[21] B. Q. Li, Q. L. Hu, X. Z. Qian, Z. P. Fang, and J. C. Shen, "Bioabsorbable chitosan/hydroxyapatite composite rod prepared by in-situ precipitation for internal fixation of bone fracture," Acta Polymerica Sinica, no. 6, pp. 828-833, 2002.

[22] W. Y. Xia, W. Liu, L. Cui, et al., "Tissue engineering of cartilage with the use of chitosan-gelatin complex scaffolds," Journal of Biomedical Materials Research Part B, vol. 71, no. 2, pp. 373380, 2004.

[23] V. Chiono, E. Pulieri, G. Vozzi, G. Ciardelli, A. Ahluwalia, and P. Giusti, "Genipin-crosslinked chitosan/gelatin blends for biomedical applications," Journal of Materials Science: Materials in Medicine, vol. 19, no. 2, pp. 889-898, 2008.

[24] Z. S. Li, H. R. Ramay, K. D. Hauch, D. M. Xiao, and M. Q. Zhang, "Chitosan-alginate hybrid scaffolds for bone tissue engineering," Biomaterials, vol. 26, no. 18, pp. 3919-3928, 2005.

[25] D. Verma, K. S. Katti, D. R. Katti, and B. Mohanty, "Mechanical response and multilevel structure of biomimetic hydroxyapatite/polygalacturonic/chitosan nanocomposites," Materials Science and Engineering C, vol. 28, no. 3, pp. 399-405, 2008.

[26] D. Verma, K. S. Katti, and D. R. Katti, "Effect of biopolymers on structure of hydroxyapatite and interfacial interactions in biomimetically synthesized hydroxyapatite/biopolymer nanocomposites," Annals of Biomedical Engineering, vol. 36, no. 6, pp. 1024-1032, 2008.

[27] D. Verma, K. S. Katti, and D. R. Katti, "Osteoblast adhesion, proliferation and growth on polyelectrolyte-complexhydroxyapatite nanocomposites," Philosophical Transactions. Series A, vol. 368, no. 1917, pp. 2083-2097, 2010.

[28] M.-W. Lee, C.-L. Hung, J.-C. Cheng, and Y.-J. Wang, "A new anti-adhesion film synthesized from polygalacturonic acid with 1-ethyl-3-(3-dimethylaminopropyl)carbodiimide crosslinker," Biomaterials, vol. 26, no. 18, pp. 3793-3799, 2005.

[29] W. Argüelles-Monal, O. L. Hechavarría, L. Rodríguez, and C. Peniche, "Swelling of membranes from the polyelectrolyte complex between chitosan and carboxymethyl cellulose," Polymer Bulletin, vol. 31, no. 4, pp. 471-478, 1993.

[30] S. M. Lim, D. K. Song, S. H. Oh, D. S. Lee-Yoon, E. H. Bae, and J. H. Lee, "In vitro and in vivo degradation behavior of acetylated chitosan porous beads," Journal of Biomaterials Science, Polymer Edition, vol. 19, no. 4, pp. 453-466, 2008.

[31] W. Tachaboonyakiat, T. Serizawa, and M. Akashi, "Inorganicorganic polymer hybrid scaffold for tissue engineeringII: partial enzymatic degradation of hydroxyapatite-chitosan hybrid," Journal of Biomaterials Science, Polymer Edition, vol. 13, no. 9, pp. 1021-1032, 2002.

[32] K. Tomihata and Y. Ikada, "In vitro and in vivo degradation of films of chitin and its deacetylated derivatives," Biomaterials, vol. 18 , no. 7 , pp. 567-575, 1997. 
[33] S. Hirano, H. Tsuchida, and N. Nagao, "N-acetylation in chitosan and the rate of its enzymic hydrolysis," Biomaterials, vol. 10, no. 8, pp. 574-576, 1989.

[34] W. L. Cao, M. Y. Cheng, Q. Ao, Y. D. Gong, N. M. Zhao, and X. F. Zhang, "Physical, mechanical and degradation properties, and schwann cell affinity of cross-linked chitosan films," Journal of Biomaterials Science, Polymer Edition, vol. 16, no. 6, pp. 791-807, 2005.

[35] D. W. Ren, H. F. Yi, W. Wang, and X. J. Ma, "The enzymatic degradation and swelling properties of chitosan matrices with different degrees of N-acetylation," Carbohydrate Research, vol. 340, no. 15, pp. 2403-2410, 2005.

[36] A. Cárdenas, W. Argüelles-Monal, F. M. Goycoolea, I. Higuera-Ciapara, and C. Peniche, "Diffusion through membranes of the polyelectrolyte complex of chitosan and alginate," Macromolecular Bioscience, vol. 3, no. 10, pp. 535-539, 2003.

[37] J. R. R. de Souza, J. I. X. de Carvalho, M. T. S. Trevisan, R. C. M. de Paula, N. Ricardo, and J. P. A. Feitosa, "Chitosancoated pectin beads: characterization and in vitro release of mangiferin," Food Hydrocolloids, vol. 23, no. 8, pp. 2278-2286, 2009.

[38] K. D. Yao, H. L. Tu, F. Cheng, J. W. Zhang, and J. Liu, "pHsensitivity of the swelling of a chitosan-pectin polyelectrolyte complex," Angewandte Makromolekulare Chemie, vol. 245, pp. 63-72, 1997.

[39] G. Balooch, G. W. Marshall, S. J. Marshall, O. L. Warren, S. A. S. Asif, and M. Balooch, "Evaluation of a new modulus mapping technique to investigate microstructural features of human teeth," Journal of Biomechanics, vol. 37, no. 8, pp. 1223 1232, 2004.

[40] R. Khanna, K. S. Katti, and D. R. Katti, "Nanomechanics of surface modified nanohydroxyapatite particulates used in biomaterials," Journal of Engineering Mechanics, vol. 135, no. 5, pp. 468-478, 2009.

[41] K. S. Katti, P. Turlapati, D. Verma, R. Bhowmik, P. K. Gujjula, and D. R. Katti, "Static and dynamic mechanical behavior of hydroxyapatite-polyacrylic acid composites under simulated body fluid," American Journal of Biochemistry and Biotechnology, vol. 2, no. 2, pp. 73-79, 2006.

[42] S. A. S. Asif, K. J. Wahl, R. J. Colton, and O. L. Warren, "Quantitative imaging of nanoscale mechanical properties using hybrid nanoindentation and force modulation," Journal of Applied Physics, vol. 90, no. 3, pp. 1192-1200, 2001.

[43] K. Kato, Y. Eika, and Y. Ikada, "In situ hydroxyapatite crystallization for the formation of hydroxyapatite/polymer composites," Journal of Materials Science, vol. 32, no. 20, pp. 5533-5543, 1997.

[44] F. Bigucci, B. Luppi, T. Cerchiara, et al., "Chitosan/pectin polyelectrolyte complexes: selection of suitable preparative conditions for colon-specific delivery of vancomycin," European Journal of Pharmaceutical Sciences, vol. 35, no. 5, pp. 435$441,2008$.

[45] D. Verma, K. Katti, and D. Katti, "Experimental inves-tigation of interfaces in hydroxyapatite/polyacrylic acid/polycaprolactone composites using photoacoustic FTIR spectroscopy," Journal of Biomedical Materials Research Part A, vol. 77, no. 1, pp. 59-66, 2006.

[46] W. W. Thein-Han and R. D. K. Misra, "Biomimetic chitosannanohydroxyapatite composite scaffolds for bone tissue engineering," Acta Biomaterialia, vol. 5, no. 4, pp. 1182-1197, 2009.
[47] H. P. Wampler and A. Ivanisevic, "Nanoindentation of gold nanoparticles functionalized with proteins," Micron, vol. 40, no. 4, pp. 444-448, 2009.

[48] J.-Y. Rho and G. M. Pharr, "Effects of drying on the mechanical properties of bovine femur measured by nanoindentation," Journal of Materials Science: Materials in Medicine, vol. 10, no. 8, pp. 485-488, 1999.

[49] S. Hengsberger, A. Kulik, and Ph. Zysset, "Nanoindentation discriminates the elastic properties of individual human bone lamellae under dry and physiological conditions," Bone, vol. 30, no. 1, pp. 178-184, 2002. 

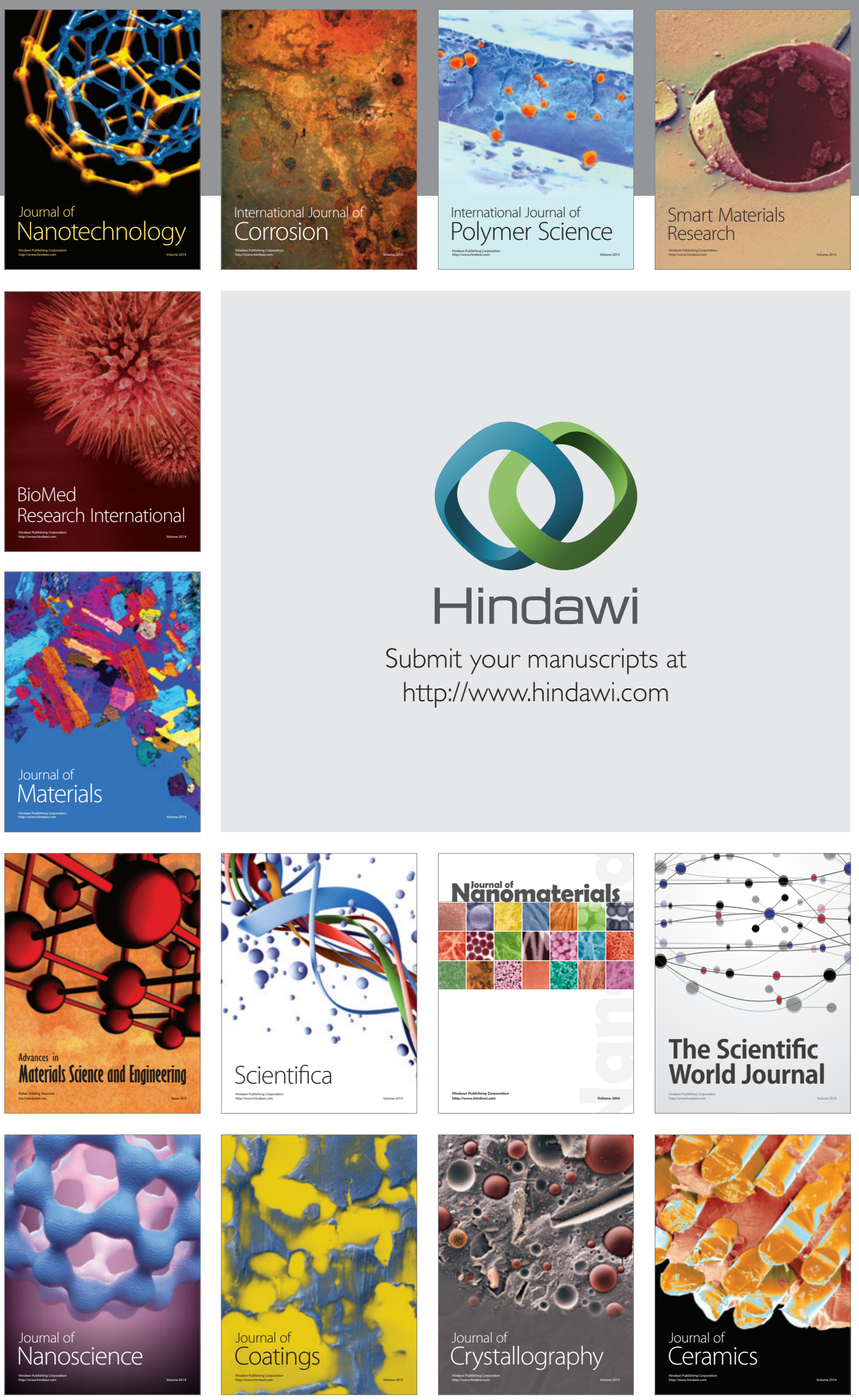

The Scientific World Journal

Submit your manuscripts at

http://www.hindawi.com

\section{World Journal}

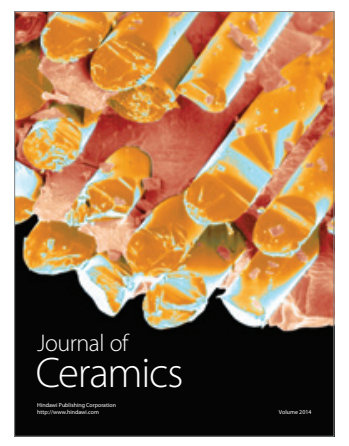

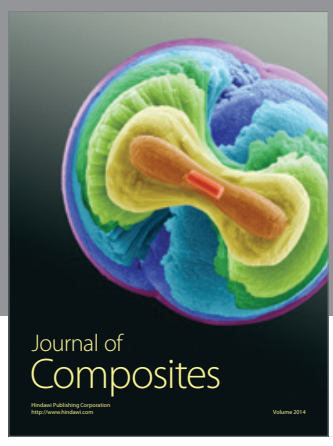
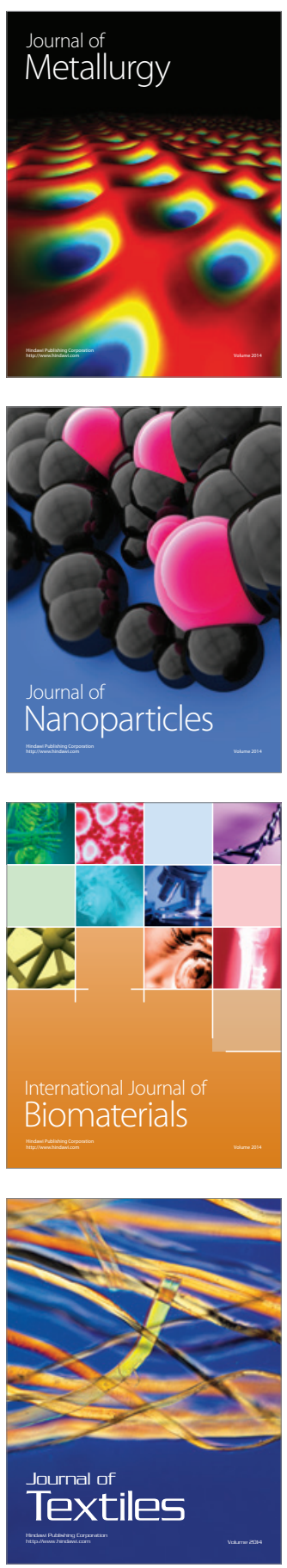